Mens

revue d'histoire intellectuelle de l'Amérique française

MENS

\title{
Québécoises deboutte! Le Front de libération des femmes du Québec, le Centre des femmes et le nationalisme
}

\section{Sean Mills}

Volume 4, numéro 2, printemps 2004

URI : https://id.erudit.org/iderudit/1024596ar

DOI : https://doi.org/10.7202/1024596ar

Aller au sommaire du numéro

Éditeur(s)

Centre de recherche en civilisation canadienne-française

ISSN

1492-8647 (imprimé)

1927-9299 (numérique)

Découvrir la revue

Citer cet article

Mills, S. (2004). Québécoises deboutte! Le Front de libération des femmes du Québec, le Centre des femmes et le nationalisme. Mens, 4(2), 183-210.

https://doi.org/10.7202/1024596ar

\section{Résumé de l'article}

La fin des années 1960 a vu la naissance du mouvement de libération des femmes au Québec. En étudiant le Front de libération des femmes (FLF) et son successeur, le Centre des femmes, nous tenterons d'expliquer les paradigmes idéologiques changeants qui ont façonné la pensée politique des deux groupes. Le FLF et, à ses débuts, le Centre des femmes avaient pour rhétorique le discours de la "libération totale ", croyant que l'émancipation des femmes était inextricablement liée à l'objectif de créer un Québec indépendant et socialiste. Cependant, au milieu des années 1970, le Centre des femmes en est venu à abandonner le nationalisme québécois et a commencé à voir dans l'émancipation de la classe ouvrière la condition nécessaire à la libération des femmes. Nous allons tenter de démontrer que le changement s'est produit en raison de la nature changeante du mouvement nationaliste comme du mouvement féministe. Alors que les femmes acquéraient de l'expérience dans l'action politique indépendante, le Parti québécois devenait de plus en plus influent au sein du mouvement nationaliste, et la valorisation de la famille nucléaire qu'on lui attribuait troublait profondément le FLF. Quand le débat sur le financement public de l'avortement est devenu fondamental pour le Centre des femmes, celui-ci a commencé à voir dans le nationalisme québécois un obstacle plutôt qu'un véhicule de la libération. Le Centre s'est dissocié du nationalisme, à l'instar d'une partie de la gauche du Québec, dans l'espoir de former un mouvement prolétaire.
Ce document est protégé par la loi sur le droit d'auteur. L'utilisation des services d’Érudit (y compris la reproduction) est assujettie à sa politique d'utilisation que vous pouvez consulter en ligne.

https://apropos.erudit.org/fr/usagers/politique-dutilisation/ 


\title{
QUÉBÉCOISES DEBOUTTE ! LE FRONT DE LIBÉRATION DES FEMMES DU QUÉBEC, LE CENTRE DES FEMMES ET LE NATIONALISME
}

\author{
Sean Mills \\ Département d'histoire \\ Université Queen's
}

\section{Résumé}

La fin des années 1960 a vu la naissance du mouvement de libération des femmes au Québec. En étudiant le Front de libération des femmes (FLF) et son successeur, le Centre des femmes, nous tenterons d'expliquer les paradigmes idéologiques changeants qui ont façonné la pensée politique des deux groupes. Le FLF et, à ses débuts, le Centre des femmes avaient pour rhétorique le discours de la « libération totale ", croyant que l'émancipation des femmes était inextricablement liée à l'objectif de créer un Québec indépendant et socialiste. Cependant, au milieu des années 1970, le Centre des femmes en est venu à abandonner le nationalisme québécois et a commencé à voir dans l'émancipation de la classe ouvrière la condition nécessaire à la libération des femmes. Nous allons tenter de démontrer que le changement s'est produit en raison de la nature changeante du mouvement nationaliste comme du mouvement féministe. Alors que 
les femmes acquéraient de l'expérience dans l'action politique indépendante, le Parti québécois devenait de plus en plus influent au sein du mouvement nationaliste, et la valorisation de la famille nucléaire qu'on lui attribuait troublait profondément le FLF. Quand le débat sur le financement public de l'avortement est devenu fondamental pour le Centre des femmes, celui-ci a commencé à voir dans le nationalisme québécois un obstacle plutôt qu'un véhicule de la libération. Le Centre s'est dissocié du nationalisme, à l'instar d'une partie de la gauche du Québec, dans l'espoir de former un mouvement prolétaire.

\section{Abstract}

The late 1960s witnessed the birth of the women's liberation movement in Quebec. By studying the Front de Libération des Femmes (FLF) and its successor, the Centre des femmes, I will attempt to explain the changing ideological paradigms that shaped the political thought of the two groups. The FLF and, at the outset, the Centre des femmes, framed its rhetoric in the discourse of "total liberation", believing that women's emancipation was inextricably linked to the goal of creating an independent and socialist Québec. By the mid-1970s, however, the Centre des femmes had abandoned Québec nationalism and began to look to working-class emancipation as the necessary condition for women's liberation. The change occurred, I will argue, because of the changing nature of both the nationalist and the women's movements. While women gained experience in independent political action, the Parti Québécois gained an increasing influence within the nationalist movement, and the party's perceived valorization of the nuclear family deeply disturbed the FLF. As the debate over publicly-funded abortion became central to the Centre des femmes, it began to see Québec nationalism as an obstacle rather than as a vehicle to liberation. Like much of the Québec left (of which it saw itself forming an important part), the Centre moved away from nationalism in the hope of forming a proletarian working-class movement.

S'inspirant de mouvements de décolonisation du TiersMonde et du féminisme radical en pleine ascension aux ÉtatsUnis, le Front de libération des femmes (FLF), alliance de 
féministes révolutionnaires tant francophones qu'anglophones, est apparu en 1969 comme un défi aux structures du pouvoir établi. Quand le groupe est disparu au bout de deux ans, le Centre des femmes a été constitué, formé en partie d'anciennes membres du FLF. Ensemble, les deux groupes ont représenté l'aube d'une nouvelle ère dans l'histoire du féminisme québécois. Pour le FLF et, à ses débuts, le Centre des femmes, la libération des femmes était inextricablement liée à l'objectif de l'indépendance du Québec. Après sa première année d'existence, le FLF est même allé jusqu'à expulser ses membres anglophones, prétextant que leur accès à la littérature féministe américaine leur assurait la maittrise de l'idéologie du groupe. Le lien entre féminisme et nationalisme radical s'est toutefois lentement dénoué au Centre des femmes et, en 1975, l'objectif de la libération nationale avait cédé la place à la lutte pour la libération des femmes par le biais de l'émancipation de la classe ouvrière.

Le mouvement féministe a été largement étudié par les spécialistes, mais la relation complexe entre féminisme et nationalisme n'a guère été abordée jusqu'à récemment ${ }^{1}$. Il n'existe aucune relation fixe entre féminisme et nationalisme et, à différents moments tout au long de leur histoire, le féminisme et le nationalisme ont été à la fois en conflit et en symbiose. Tentant d'examiner la relation changeante du mouvement de libération des femmes avec le nationalisme québécois, nous avons étudié les documents du FLF et du Centre des femmes. En analysant les documents internes, les documents de presse et les publications des deux organisations, nous espérons démontrer la nature complexe de leur idéologie changeante. 


\section{Le FLF, le Centre des femmes et la libération nationale}

Les années 1960, tant au Québec que dans tout le monde occidental, ont été une période de grande ébullition. Du mouvement des droits civiques aux États-Unis à celui de mai 68 à Paris, les démocraties occidentales ont été profondément remises en question par une myriade de mouvements dissidents. Au Québec, sécularisation et modernisation à grande échelle ont transformé les institutions provinciales. La dislocation sociale des années 1960 a aussi eu une incidence importante sur les Québécoises. Micheline de Sève a écrit : «Les femmes illustrèrent massivement par leur comportement qu'elles entendaient dorénavant s'affirmer elles aussi, maittres de soi. » À partir de 1961, ajoute-t-elle, le taux de natalité a diminué, les naissances hors mariage ont augmenté de façon spectaculaire et les femmes mariées en sont venues à jouer un plus grand rôle au sein de la population active ${ }^{2}$. En 1966, le tiers des femmes de 24 à 34 ans avaient un emploi rémunéré, mais ce chiffre atteignait $39,9 \%$ en 1971 et a continué de grimper par la suite. Les Québécoises ont obtenu bien des gains, par exemple les progrès législatifs contenus dans la loi omnibus de Pierre Trudeau en 1969, mais les effets de la Révolution tranquille en ce qui concerne les femmes demeurent nettement mitigés. Les femmes s'intégraient dans la population active en nombres records, mais elles se trouvaient marginalisées dans des "professions féminines » et on s'attendait souvent à ce qu'elles aient une double occupation, effectuant un travail rémunéré le jour et s'occupant des tâches ménagères le soir ${ }^{3}$. Et, comme le montre Micheline Dumont, les femmes occupaient un grand nombre de postes d'autorité dans les domaines de l'éducation, des services sociaux et des soins hospitaliers dont elles ont été graduellement exclues après $1960^{4}$. 
De vastes changements sociaux se produisant autour d'elles, les Québécoises ont commencé à s'organiser afin de pouvoir participer aux changements de la Révolution tranquille. En 1965, la Fédération des femmes du Québec (FFQ) a été fondée, marquant le début de la seconde période du mouvement féministe québécois ${ }^{5}$. Ce n'est que quelques années après la fondation de la FFQ, dans un contexte de grand bouleversement social, que le FLF est né, soit en 1969. Un groupe de femmes, le Front commun des Québécoises, est descendu dans les rues pour protester avec colère contre un règlement municipal restreignant les manifestations publiques dans les rues de Montréal. Portant des chaînes pour symboliser leur répression, les femmes ont défilé pendant la nuit et 165 des 200 protestataires ont été arrêtées par la police municipale $^{6}$. D'après Solange Chalvin, journaliste au Devoir, c'était un « mouvement spontané né de l'indignation d'un groupe de jeunes femmes ${ }^{7}$ ». Après la protestation, un groupe de femmes a décidé de fonder le FLF. Un nombre à peu près égal d'anglophones et de francophones est à l'origine de la fondation du groupe, mais ce sont les anglophones, influencées par le féminisme américain ${ }^{8}$, qui ont poussé les francophones à se lancer dans une action politique indépendante. Selon Martine Lanctôt, un écart important séparait les motivations des féministes francophones et anglophones :

Les femmes [francophones] qui sont le plus susceptibles d'adhérer au mouvement de libération des femmes sont justement celles-là mêmes qui sont impliquées dans les luttes politiques de l'époque et qui sont engagées dans le mouvement nationaliste. Elles sont alors peu concernées par la lutte de libération des femmes. Ce sont les féministes anglophones de Montréal qui vont inciter les francophones à participer à la lutte de libération des femmes?. 
Après bien des discussions, le FLF s'est donné pour objectif la libération des femmes par la création d'un Québec indépendant et socialiste.

Les femmes anglophones étaient influencées par le mouvement américain de libération des femmes, tandis que les francophones, pour la plupart, se détachaient des mouvements de gauche dominés par les hommes, lesquels mouvements, selon elles, ne les prenaient pas au sérieux et ne tenaient pas compte de leurs besoins. Dans les groupes mixtes, le FLF soutenait que les femmes étaient reléguées à des rôles subordonnés et traditionnellement "féminins » tels que la dactylographie et la préparation du café. Pour de " true revolutionaries ", faisait observer sarcastiquement le FLF, il y a " so many other things ... to do besides worry about women ». " Radicals have always had, and still have, the time to go out and get stoned on weekends, but not the time to create day nurseries, to organize domestic brigades, without which women cannot be expected to make the revolution ${ }^{10}$ ». Outre qu'elles étaient reléguées aux " tâches féminines » au sein des groupes révolutionnaires mixtes, Stéphanie Lanthier a démontré que les femmes étaient symboliquement exclues du mouvement nationaliste radical. Le nationalisme québécois radical, soutient-elle, a construit " son modèle de libération nationale sur la soumission et l'exclusion des femmes ". Lanthier affirme que "[] bolisées par le pays ou par la culture anglo-saxonne, elles sont absentes de toute analyse historique ${ }^{11} »$. La création d'organisations féminines autonomes, croyaient les militantes, était une étape cruciale vers la réalisation d'une véritable « révolution dans la révolution ${ }^{12}$ ».

La structure organisationnelle du FLF était composée de plusieurs cellules indépendantes et le groupe espérait mettre de l'avant une "solidarité qui nous permettra d'élaborer 
ensemble ce que devra être notre libération ${ }^{13}$ ". Même si le FLF (et plus tard le Centre des femmes) espérait rejoindre la classe ouvrière, le groupe en soi était largement composé de femmes relativement privilégiées. Selon Véronique O’Leary, ancienne militante, " elles avaient au moins une instruction secondaire, ce qui leur permettait de travailler dans le domaine tertiaire (les services, la culture) ». Ce qui les a poussées au féminisme, dit O’Leary, a été le contraste énorme entre théorie et réalité : "Théoriquement, nous étions les égales des hommes, mais pratiquement, nous demeurions toujours inférieures $^{14}$. ”

À ses débuts, le FLF a lutté pour la libération nationale du Québec, laquelle était, croyait-il, la condition de l'émancipation des femmes. Le mot d'ordre du FLF révélait clairement les principes premiers de son idéologie : "PAS DE LIBÉRATION DU QUÉBEC SANS LIBÉRATION DES FEMMES, PAS DE LIBÉRATION DES FEMMES SANS LIBÉRATION DU QUÉBEC. " Le groupe situait donc sa lutte dans la rhétorique de la libération totale. "La libération de la femme, lisait-on dans un bulletin du FLF, n'a pas pour but d'en faire l'oppression d'autres groupes d'individus, mais elle s'insère dans un processus de libération de tous les êtres humains. Il ne faut pas inverser les rôles mais les changer ${ }^{15}$. " Avec son objectif de libération totale, le FLF s'inspirait de groupes révolutionnaires dominés par des hommes, et tant son nom que son organisation interne étaient consciemment liés au Front de libération du Québec. ${ }^{16}$

Malgré l'importance qu'il accordait à la rhétorique de la libération nationale, le FLF ne peut être considéré comme un simple appendice du mouvement révolutionnaire à dominante masculine. Selon Lanthier, "les paradigmes du mouvement de libération des femmes s'établissent dans l'idée d'une révolution globale des structures de rapports entre les hommes et 
les femmes ${ }^{17}$ ». L'émancipation des femmes n'était pas considérée comme une conséquence de l'indépendance du Québec, mais elle était plutôt vue comme une des conditions nécessaires à un mouvement révolutionnaire véritablement national. Le FLF déclarait : "Nous considérons que les femmes ne pourront se libérer qu'à l'intérieur d'un processus de libération globale de toute la société. Cette libération ne sera possible qu'avec la participation entière et à tous les niveaux des femmes qui composent la moitié de la population québé$\operatorname{coise}^{18}$.» En remettant en question les relations entre les sexes au sein du mouvement révolutionnaire global, les membres du FLF espéraient assurer les conditions d'une émancipation humaine totale. Il était impérieux que les féministes se battent pour l'émancipation des femmes, mais les Québécoises ne pouvaient non plus oublier qu'il leur fallait se joindre à «la lutte de libération nationale du peuple québécois, sans quoi, cette libération ne serait qu'illusoire ${ }^{19} \%$.

Tout en empruntant le langage de la libération nationale, les membres du FLF situaient également leur mouvement dans un contexte international de décolonisation. Dans le Québec des années 1960, beaucoup, à gauche, reliaient la lutte québécoise pour la libération nationale aux mouvements du Tiers-Monde contre l'impérialisme. " [L]es féministes québécoises de la première heure, soutient O'Leary, étaient plus tiers-mondistes qu'ailleurs : on se sentait très près des femmes des mouvements de libération du tiers-monde ${ }^{20} »$. D'après Diane Lamoureux, le féminisme québécois à la fin des années 1960 et au début des années 1970 était très influencé par de nombreuses tendances internationales. D'une part, affirme-telle, les féministes étaient influencées par le marxisme, et d'autre part, elles modelaient leurs combats sur les mouvements de décolonisation. Le langage de la décolonisation était " la meilleure garantie de contenu "authentiquement révolu- 
tionnaire" que pourrait se donner le mouvement féministe ${ }^{21}$ ». Se situant dans un contexte international, les femmes du FLF tentaient de mettre leur lutte sur le même plan que d'autres mouvements de libération. On pouvait lire dans un bulletin du FLF : "Cette situation [d'exploitation] est la même pour tous les groupes humains exploités : les Noirs, les Québécois, les colonisés à travers le monde ${ }^{22}$. " La conviction que le Québec était une nation tout à fait colonisée ayant besoin d'être libérée était au cœur de toutes les analyses du FLF concernant les femmes au Québec. Pour Lamoureux, « l'assimilation de l'oppression de la nation québécoise à une situation coloniale $^{23}$ » était le point de départ de l'analyse sociale du FLF.

Étant donné que les Québécoises étaient exploitées au niveau national par la colonisation, sur le plan économique par le capitalisme et sur le plan social par le patriarcat, il était impératif qu'une révolution totale ait lieu pour transformer toutes les structures sociales. Pour le FLF, l'exploitation économique des Québécoises était « directement influencée par l'impérialisme américain (importance du secteur tertiaire, économie sous-développée), par le colonialisme anglo-saxon et par le capitalisme ${ }^{24} »$. Dans son Bulletin de Liaison de 1971, le FLF définissait clairement la nature interdépendante de son programme. D'après ses auteurs, le groupe luttait :

Pour l'indépendance, parce que nous sommes femmes mais femmes québécoises et en tant que Québécoises nous sommes colonisées. Pour le socialisme, parce que même si l'exploitation de la femme est antérieure au capitalisme et même si cette exploitation ne se fait pas selon un mode capitaliste (plutôt selon le mode du servage), nous vivons quand même dans un système capitaliste et c'est ce système-là qui utilise l'exploitation de la femme aujourd'hui ${ }^{25}$. 
Pour se libérer de l'exploitation nationale, sexuelle et capitaliste, les femmes du FLF croyaient qu'il était nécessaire pour les féministes de lutter au sein d'organisations féminines indépendantes préconisant la libération nationale par la révolution socialiste.

Les femmes du FLF avaient une relation trouble avec les groupes activistes anglophones. Le groupe a par exemple refusé de participer à la caravane de l'avortement vers Ottawa organisée par des anglophones. Dans un communiqué de presse de mai 1970, le FLF expliquait ainsi son refus de participer :

Camarades, nous refusons d'aller manifester devant un parlement dont nous ne reconnaissons pas les pouvoirs qu'il s'arroge sur le Québec. Cependant, nous sommes solidaires des femmes du Canada, puisque étant femmes, nous subissons la même oppression... Nous avons les mêmes aspirations : nous voulons faire passer la terre de la fatalité à la libertée

En refusant de participer à la première manifestation pancanadienne du mouvement de libération des femmes ${ }^{27}$, les membres du FLF tentaient de démontrer avec éclat que le nationalisme québécois était au cœur de leur cause.

En plus de se distancier des organisations féministes du Canada anglais, le FLF a décidé, à l'automne 1970, d'exclure les anglophones de ses rangs. D'après Lanctôt, "[l]'importance accordée par les francophones à la lutte de libération nationale est à l'origine des intérêts divergents et des conflits qui vont se manifester rapidement entre les deux groupes linguistiques ${ }^{28} »$. Véronique O'Leary et Louise Toupin montrent comment les femmes francophones du FLF s'inquiétaient du déséquilibre qui existait entre les membres francophones et anglophones du groupe. C'est au cours d'une réunion de deux jours dans les Laurentides, rappellent-elles, que 
les membres francophones du groupe ont décidé d'exclure les anglophones. Elles ajoutent : "Parmi les raisons invoquées, mentionnons celles voulant que les anglophones, du fait qu'elles ont accès à toute la documentation américaine et anglaise sur le "Women's Lib.", exercent un contrôle idéologique sur le FLF, imprimant ainsi au groupe une tendance américaine, sans égard à la réalité québécoise. " L'existence d'un corps croissant de textes féministes en langue anglaise provenant des États-Unis était donc perçue comme une menace par les membres francophones du FLF. Pour ces dernières, «[]]a transmission de leurs connaissances [celles des anglophones] sur ce qui se passait outre-frontière et leurs opinions leur semblaient, de ce fait, relever d'une attitude toute colonisatrice ». Espérant créer un mouvement basé sur une sensibilisation à l'échelle internationale à l'oppression que subissent les femmes et sur une solide compréhension de problèmes spécifiques aux Québécoises, le FLF croyait que le mouvement ne devait être formé que de francophones. Les anglophones du groupe, qui représentaient la moitié du nombre total des membres, ont été choquées par leur exclusion de l'organisation qu'elles avaient contribué à former. Nombre d'anglophones, affirment O'Leary et Toupin, "très intégrées à la communauté francophone et indépendantistes, par surcroit, ne "digèrent" pas l'exclusion et arrêtent toute activité féministe ${ }^{29}$ ".

Peu après l'expulsion des membres anglophones du FLF, en décembre 1970, le groupe a décidé de refuser de participer à une conférence sur la libération des femmes organisée par des Américaines qui devait avoir lieu à Montréal. Bien qu'ayant, au début, manifesté de l'intérêt pour l'idée, le FLF s'y est opposé en raison des troubles politiques et du climat de peur créé par l'application de la Loi sur les mesures de guerre à peine quelques mois auparavant. Dans sa lettre aux féministes américaines, le FLF a clairement défini sa position 
relativement aux féministes anglophones à Montréal et ailleurs. Si la conférence était organisée à Montréal par des féministes anglo-canadiennes ou américaines, le FLF affirmait : « [N]ous interprétons inévitablement ce geste comme une autre manifestation du colonialisme que nous subissons quotidiennement. " La lettre éclaircissait ensuite la position du FLF sur Marlene Dixon, le contact montréalais des féministes américaines : " Marlene est professeur à l'Université McGill (anglophone) ; elle n'appartient ni au Women's Lib de Montréal, ni au Front de libération des femmes du Québec. Elle vit ici depuis un an et demi et elle n'a jamais pu communiquer directement avec nous car elle ne parle toujours pas français. Elle ne peut donc en aucune façon parler en notre nom ni en celui d'aucune Québécoise ${ }^{30}$. » En excluant ses membres anglophones et en se distanciant clairement des organisations féministes de langue anglaise, le FLF démontrait, croyait-il, l'importance du nationalisme dans son idéologie.

Après deux années turbulentes passées à lutter pour libérer le Québec de la domination capitaliste coloniale, le FLF avait organisé de nombreuses manifestations, ouvert une garderie et publié la première édition d'un journal. Il n'avait jamais attiré beaucoup d'adeptes, ne dépassant probablement jamais une soixantaine de membres organisés en cellules indépendantes, mais il a eu une incidence importante sur le mouvement féministe québécois ${ }^{31}$. En 1971, des divisions internes et une diminution radicale du nombre des membres ont découragé les quelques militantes qui restaient, lesquelles ont décidé de dissoudre le groupe. Quand le FLF est disparu, l'idée de la triple exploitation, sexuelle, nationale et sociale, des Québécoises s'est intégrée, plus ou moins directement, au cadre théorique du Centre des femmes. Quand ce dernier a été créé en janvier 1972, il voulait offrir un forum de sensibilisation et d'étude du statut des femmes. Le FLF et 
le Centre des femmes ne peuvent en aucune façon être traités comme une entité unique, mais les femmes qui formaient le Centre des femmes se considéraient dans une certaine mesure comme œuvrant dans la continuation logique du $\mathrm{FLF}^{32}$. En donnant à leur journal, Québécoises Deboutte !, le même titre que celui utilisé par le FLF, et en conservant le même slogan, les membres du Centre des femmes cherchaient consciemment à établir un lien entre les deux mouvements.

Contrairement à Martine Lanctôt, qui soutient que, dès le début, les "positions [du Centre] sur la question nationale seront $[\ldots]$ beaucoup plus nuancées ${ }^{33} "$, les premiers documents du Centre soulignent clairement sa foi dans la libération nationale. Dans le premier numéro de Québécoises Deboutte! du Centre des femmes, le groupe affirmait nettement adhérer aux principes originels du FLF. D'après ce périodique, "la libération des femmes n'est pas une libération individuelle ou culturelle : la lutte de libération des femmes doit se faire dans le cadre de la libération nationale, sociale, économique, politique et culturelle ». Il était donc nécessaire de situer "[leur] rôle de ménagères, de travailleuses et de reproductrices dans le contexte de la société québécoise $e^{34} »$. En outre, le Centre des femmes continuait de privilégier la classe ouvrière québécoise et veillait à ce que son analyse de la société se fasse dans un cadre québécois.

Comme la lutte "implique nécessairement comme objectif premier un changement radical de la société québécoise », le Centre des femmes cherchait à définir « les termes de notre oppression, préciser nos objectifs dans la conjoncture actuelle de la lutte du peuple québécois, décider de nos moyens de lutte, travailler à rassembler les Québécoises dans une organisation révolutionnaire qui sera leur force ». À cette fin, le Centre des femmes s'est servi de son journal comme d'un forum permettant l'expression d'un récit historique s'op- 
posant aux représentations dominantes du passé qui ignoraient les contributions des femmes du Québec. Étant donné que les historiens « sont particulièrement intéressés au passé de la classe dominante blanche et masculine $e^{35} »$, il était nécessaire de récupérer la voix des femmes du passé. Dans la première édition de Québécoises Deboutte!, les femmes du Centre soulignaient fermement la nécessité d'offrir une version révisionniste de l'histoire du Québec :

Pour nous, femmes québécoises, l'histoire n'est pas encore écrite. Les tentatives récentes de retracer le passé d'oppression du peuple québécois ont encore "négligé » de souligner le nôtre. Pourtant, même si l'histoire des Québécoises suit les grandes lignes définies par un contexte économique, politique et social identique, il reste que nous avons vécu une oppression spécifique due aux rôles qui nous sont dévolus dans une société patriarcale ${ }^{36}$.

Le Centre a donc lancé un projet visant à offrir, par une série d'articles, une autre histoire du Québec. Dans le but de présenter « l'oppression spécifique des femmes québécoises dans l'histoire de notre peuple » et de détruire " les mythes qui nous empêchent à l'heure actuelle de mieux lutter parce que nous comprenons mal les cadres de notre oppression ", le groupe a réinterprété l'histoire des sociétés amérindiennes et réexaminé des événements tels que la Conquête et les Rébellions du Bas-Canada du point de vue des femmes ${ }^{37}$.

En plus de réécrire l'histoire du Québec, le Centre des femmes, à ses débuts, s'est consciemment dissocié d'autres groupes féministes moins radicaux et moins nationalistes. Par exemple, le Centre a rejeté la fondation de l'Association féministe de Montréal, groupe qui avait pour objectif de réunir le plus grand nombre de femmes possible dans une lutte commune en faveur des droits des femmes. Le Centre s'opposait à 
l'union de femmes de tous horizons idéologiques et croyait que toute tentative d'intégrer des femmes de différentes classes sociales dans le mouvement nuirait à la tentative de susciter une authentique conscience de classe. La tentative d'organiser les femmes avant qu'elles n'aient pris conscience de leur oppression, croyait le Centre, "ne peut que regrouper entre elles des femmes privilégiées qui sont par le fait même coupées des intérêts de la majorité des femmes québécoises, travailleuses et ménagères ${ }^{38}$ \%. Un autre problème important posé par l'Association féministe de Montréal était que ses réunions, tout en étant ostensiblement bilingues, prenaient "comme de coutume l'allure d'une rencontre unilingue anglaise. D'ailleurs l'assistance était $85 \%$ anglophone ${ }^{39} »$. Le Centre considérait donc l'Association féministe de Montréal comme une organisation réformatrice qui ne comprenait pas véritablement la nécessité de la libération nationale.

Pour le FLF et, à ses débuts, le Centre des femmes, la lutte pour la libération nationale était un élément central de tout projet visant à libérer véritablement les femmes du Québec. Cependant, le mouvement devenant plus mature, de nombreuses féministes québécoises ont commencé à se méfier du mouvement nationaliste, de plus en plus dominé par le Parti québécois $(\mathrm{PQ})$.

\section{Le déclin du nationalisme}

Le Centre des femmes est né de la dissolution du FLF et, à ses débuts, est demeuré loyal aux principes nationalistes de ce dernier, mais le nationalisme du groupe a lentement décliné. Au début, le mouvement féministe avait acquis une expérience importante de l'action politique indépendante et il a commencé à abandonner dans une certaine mesure la thétorique nationaliste qui avait imprégné sa première orientation idéologique. En même temps, le Centre a décidé de s'en- 
gager pleinement dans le combat pour la légalisation de l'avortement et son financement par l'État, et le groupe a commencé à confondre nationalisme et politiques du PQ. Comme le groupe s'opposait énergiquement à ce qu'il tenait pour la glorification par le PQ de la maternité et de la famille nucléaire, le Centre en est venu à rejeter entièrement le projet nationaliste.

Au début des années 1970, le Centre des femmes a augmenté spectaculairement sa participation à la lutte en faveur de la légalisation de l'avortement et de son financement par l'État, combat qu'il considérait comme une conséquence naturelle du désir d'éliminer le capitalisme. L'avortement avait été, depuis la formation du FLF, un élément important du programme du mouvement de libération des femmes, mais il devenait maintenant un des principes centraux de l'idéologie du Centre des femmes. D'après Diane Lamoureux, le FLF, croyant que l'avortement serait légalisé au Québec dans un avenir proche, pensait qu'il serait plus utile de se concentrer sur autre chose ${ }^{40}$. À l'opposé du FLF, le Centre des femmes en viendrait à jouer un rôle central dans la lutte en faveur de la légalisation de l'avortement.

C'est l'arrestation du $\mathrm{D}^{\mathrm{r}}$ Morgentaler qui a incité pour la première fois le Centre à s'engager activement dans le combat, mais il n'a pas limité sa lutte à la défense des droits de ce médecin. "Nous ne voulons pas, déclarait-il, concentrer la lutte uniquement autour de la défense de médecins. Il ne s'agit pas de défendre le droit des médecins à pratiquer des avortements, mais de s'assurer à nous les femmes, le droit d'avorter librement et gratuitement ${ }^{41} »$. L'avortement était, non une fin en soi, mais une étape cruciale vers l'émancipation féminine. Dans un article de Québécoises deboutte!, une auteure précise la position du Centre : 
Pour nous, la légalisation de l'avortement (avortement gratuit et sur demande) n'est pas une fin en soi ; cependant c'est un service essentiel que doit nous rendre une société qui n'assure même pas les conditions matérielles minimum nous permettant d'élever des enfants (garderies gratuites, congés de maternité payés, collectivisation des travaux ménagers, etc.). Pour nous les femmes, le contrôle des naissances par la contraception et l'avortement (si la contraception a fait défaut) est primordial. C'est le premier pas vers une possibilité de prise en main de nos vies ${ }^{42}$.

Outre qu'il préconisait la légalisation de l'avortement et son financement par l'État, le Centre voyait dans sa criminalisation un corollaire du capitalisme. "On ne peut séparer la politique d'un pays sur l'avortement, soutenait le groupe, de sa politique générale en matière de lois économiques et sociales. " $\mathrm{La}$ question de l'avortement, poursuivait le Centre, " est intimement reliée aux conditions sociales, économiques et politiques d'un pays ». Au Québec, deux groupes d'intérêts principaux débattaient de la question : l'État et l'Église d'une part, et, d'autre part, les femmes de la classe ouvrière ${ }^{43}$. Parce qu'une partie du rôle des femmes dans le système capitaliste était la reproduction dans le but de garantir la prochaine génération de travailleurs, croyait le Centre, la lutte pour l'avortement « remet en cause un des fondements du système capitaliste ${ }^{44}$ ».

En 1973, le Centre a annoncé qu'il participerait à la lutte pour la légalisation de l'avortement. Établissant clairement un lien entre le capitalisme et la famille nucléaire, le Centre a rejeté sa décision antérieure de s'abstenir de participer au débat sur l'avortement. Le groupe déclarait fièrement : "Le Centre des femmes [prend] position clairement CONTRE LA RÉPRESSION DU GOUVERNEMENT CAPITALISTE, POUR L'OBTENTION DE L'AVORTEMENT GRATUIT ET SUR DEMANDE. » Le groupe considérait 
l'avortement comme " un problème politique d'importance ", mais il avait jusque-là refusé "d'engager la lutte ${ }^{45}$ ». Mais maintenant le Centre décidait qu'il n'était plus en mesure de rester sur " la défensive et d'attendre un cadeau du gouvernement. Car il n'y aura pas de cadeau ${ }^{46} »$. En 1974, le Centre s'était joint à d'autres groupes aux vues similaires pour former le Comité pour l'avortement libre et gratuit ${ }^{47}$.

Au moment où le Centre des femmes en venait à s'impliquer fortement dans la lutte pour la légalisation de l'avortement, la gauche québécoise francophone vivait une transition majeure. Le choc de la Crise d'octobre de 1970 et la répression policière subséquente ont amené plusieurs défenseurs de la libération nationale révolutionnaire à repenser leur stratégie politique. Au début des années 1970, selon Paul-André Linteau et al., la gauche québécoise avait une relation ambiguë avec le nationalisme ${ }^{48}$. Lorsque Pierre Vallières, l'un des principaux théoriciens du mouvement de libération nationale, a publié un essai annonçant sa décision de joindre le $\mathrm{PQ}$, cela aurait eu «l'effet d'une véritable bombe et eut des retombées considérables au sein du mouvement québécois de libération nationale. Une page d'histoire était définitivement tournée, celle du F.L.Q. et de la violence révolutionnaire ${ }^{49} »$. Alors que Vallières et plusieurs autres décidaient de se dissocier de l'activité révolutionnaire en joignant le $\mathrm{PQ}^{50}$, un nombre considérable d'acteurs politiques s'est tourné vers le marxisme-léninisme espérant créer un véritable parti prolétaire ${ }^{51}$. Dû à la dispersion des nationalistes radicaux des années 1960 choisissant des voies divergentes, le Parti québécois, gagnant en popularité, s'est mis à occuper de plus en plus l'espace idéologique relatif à la question nationale ${ }^{52}$.

Comme les militantes du Centre des femmes se sont toujours considérées comme une partie intégrante d'un mouvement social plus large, la réorientation de la gauche québé- 
coise au début des années 1970 les a forcées à choisir leur priorité : l'émancipation de la classe ouvrière ou l'indépendance nationale. En raison de l'influence du nationalisme canadien-français traditionnel et de l'importance qu'il accordait à la survie culturelle, les féministes ont toujours eu, selon Diane Lamoureux, une relation plutôt tendue avec le $\mathrm{PQ}^{53}$. De nombreuses féministes ont cherché à mettre en lumière la nature exploitante de la famille nucléaire, mais, pour les nationalistes québécois des années 1970, « la famille n'est pas seulement l'unité de base de la société, c'est le microcosme de la nation ». Et, comme on peut s'y attendre, «dans le cadre d'une conception de la famille comme microcosme de la nation, le rôle de la mère est central ${ }^{54}$ ». Dans une série d'articles accablants, le Centre a attaqué férocement le PQ pour ses solutions illusoires visant à améliorer la condition des femmes dans la province. L'élection de quelques femmes «à l'exécutif et la série de mesures "pro-féminines" adoptées au congrès du P.Q. (fin février) ne nous apparaissent être qu'un masque trompeur ». Le PQ, accusait le Centre, désirait voir « une bourgeoisie québécoise alliée à l'impérialisme américain se développer et prendre pouvoir " et faisait miroiter de faux espoirs tant aux ouvriers qu'aux femmes. Le parti ne leur donnait que « des miettes pour les rallier autour de "l'unité nationale" 55 ».

Aux yeux du Centre, l'éloge de la famille nucléaire par le parti et, par voie de conséquence, de la domestication féminine était encore plus inquiétant que les politiques du PQ à l'égard des ouvriers et des femmes. « La mesure visant à distribuer des primes à la natalité, soi-disant "en reconnaissance de l'apport de la mère à la société", soutenait le Centre, a elle aussi pour but d'institutionnaliser ce travail privé effectué dans le cadre de la famille. " En outre, au lieu de "valoriser la femme en tant qu'individu, c'est la femme-mère que l'on glo- 
rifie ». Le Centre des femmes, comme il fallait s'y attendre, liait aussi la préservation de la famille souhaitée par le PQ au capitalisme. Pour ce groupe, " [1]a restauration de la famille en tant qu'assise idéologique du capitalisme et du pouvoir autoritaire de l'État a toujours constitué un point crucial de la politique culturelle des partis nationalistes de droite. Le P.Q. ne saurait être une exception ${ }^{56}$. »Comme le Centre des femmes voyait dans la famille nucléaire une institution étouffante, la valorisation par le PQ de la structure familiale était considérée comme un obstacle dans la lutte pour la libération des femmes. Il était donc évident pour elles que « derrière la phraséologie péquiste du "conjoint au foyer" se retrouve toute l'idéologie nationaliste du "retour au foyer" avec l'éternelle revalorisation du rôle de mère-épouse $e^{57}$ \%.

Après s'être violemment opposé au PQ, le Centre a commencé à se distancier du projet nationaliste en général. Le nationalisme québécois, semblait-il, n'était plus compatible avec la lutte du Centre pour la légalisation de l'avortement et son financement par l'État. La rhétorique de l'oppression nationale disparaissant lentement, le Centre s'est de plus en plus focalisé sur l'exploitation des classes. Remplaçant son mot d'ordre antérieur liant la libération du Québec à la libération des femmes, le Centre des femmes a adopté en 1973 un slogan davantage orienté vers les classes et moins nationaliste : "PAS DE LIBÉRATTON DES MÉNAGËRES SANS LIBÉRATION DES TRAVAILLEURS, PAS DE LIBÉRATION DES TRAVAILLEURS SANS LIBÉRATION DES MÉNAGÈRES ${ }^{\beta}{ }^{8}$ "S'intéressant dorénavant au problème des classes, le Centre a commencé à se considérer comme participant à la lutte internationale de l'ensemble des femmes et des travailleurs. C'est en faisant « de la libération des femmes une revendication de la classe ouvrière que nous réussirons à mettre fin au système d'exploitation actuel ${ }^{59} \%$. Les fem- 
mes constituaient un élément essentiel, quoique non rémunéré et peu estimé, du mode capitaliste de production. En effectuant les tâches ménagères, les femmes, entre autres choses, reproduisaient " la force de travail du mari ». Le travail non rémunéré des ménagères constituait donc «le ciment économique du système capitaliste ». Dans une société où la valeur humaine est déterminée par l'argent, " la femme se retrouve donc exclue de tous les centres de décision ${ }^{60}$ ». Quand, en 1974, le Centre s'est penché sur ses deux premières années d'activité, il a annoncé que, pour lui, le féminisme était « la lutte révolutionnaire contre l'exploitation des femmes, plus spécifiquement celles de la classe ouvrière, dans le système capitaliste $^{61} »$. Au milieu des années 1970, c'est l'émancipation de la classe ouvrière, plutôt que l'indépendance nationale, qui devenait la condition nécessaire de la libération des femmes.

En plus de privilégier désormais la classe ouvrière, et non plus l'oppression nationale, les femmes du Centre des femmes ont commencé à se considérer comme prenant part à une lutte internationale pour la libération des femmes. Au lieu de chercher dans d'autres pays une inspiration pour la décolonisation, le Centre se voyait partie d'un mouvement s'étendant bien au-delà des frontières du Québec. Tant dans les écrits publiés dans Québécoises deboutte! que dans les documents internes du Centre, le combat contre l'exploitation spécifique du Québec a cédé la place à une lutte internationale contre l'exploitation des femmes. Dans chaque partie du globe, déclarait le groupe, "il y a des millions de femmes de toutes races, de toutes nationalités qui subissent la même discrimination et exploitation ${ }^{62}$ ». Le Centre des femmes s'est mis à publier dans Québécoises deboutte! des récits de luttes de femmes à travers le monde. La capacité des Chinoises de se libérer à la fois du capitalisme et du patriarcat, par exemple, était 
particulièrement louée ${ }^{63}$. Quand Mariarosa Dalla Costa et Selma James, deux féministes-marxistes, sont venues à Montréal en avril 1973, le Centre a organisé une conférence publique en leur honneur et Québécoises debontte! a traduit et publié une entrevue de quatorze pages avec ces femmes ${ }^{64}$. Les féministes révolutionnaires du Québec en étaient venues à accepter chaleureusement des militantes internationales, considérées maintenant comme des collègues dans un combat commun.

\section{$* * *$}

À la fin de 1974, quand le Centre a réalisé une évaluation interne de ses activités, il a admis se considérer comme " un noyau féministe d'avant-garde ayant un rôle de "direction" à jouer au sein du mouvement féministe ». Le problème était cependant que « les bases mêmes pour la création de ce mouvement n'existaient pas encore ${ }^{65} »$. Miné par des divisions et des conflits internes, le Centre des femmes s'est dissout juste au moment où le mouvement féministe entrait dans une nouvelle phase de pensée et d'action ${ }^{66}$.

Si la formation du FLF en 1969 a marqué le début d'une nouvelle période de l'histoire du féminisme au Québec, il était évident en 1975 que cette période était terminée. Les $\mathrm{Na}$ tions-Unies ont déclaré 1975 l'Année internationale des femmes et le mouvement féministe a pris un nouvel élan tandis qu'un nombre sans précédent de femmes adhéraient à la cause. À partir de 1975, d'après le Collectif Clio, les femmes francophones, libres de l'emprise du marxisme et du nationalisme, adoptèrent une forme de féminisme plus autonome ${ }^{67}$. Évoquant son expérience au sein du FLF et du Centre des femmes, Véronique O'Leary a cependant parlé de l'importance pour les premières féministes du mouvement de libération des femmes d'avoir recours aux normes et aux modèles de libéra- 
tion déjà établis par la gauche québécoise. Le groupe, a-t-elle déclaré, " n'arrivait pas à se définir en dehors d'une certaine norme de gauche : on ne pouvait pas en sortir parce que c'était là.... Mais à l'époque aussi, tout le monde était tellement contre nous, qu'on avait besoin, je pense, de ces normes-là $\grave{l}^{68} \%$. Les années postérieures à 1975 ont vu une prolifération et une diversification sans précédent des organismes féministes au Québec. Le FLF et le Centre des femmes ont donné à leurs héritières le courage de s'exprimer à la fois au sein du mouvement nationaliste et contre lui.

Traduction : Christian Bérubé

\section{NOTES}

${ }^{1}$ Il n'est pas surprenant que, parmi les féministes canadiennes, les universitaires québécoises aient réalisé les études les plus intéressantes sur l'interaction entre nationalisme et féminisme. Diane Lamoureux a fait l'effort le plus soutenu pour comprendre la relation entre les projets nationaliste et féministe. Voir, par exemple, Diane Lamoureux, « Nationalisme et féminisme : impasse ou coïncidences », Possibles, vol. 8, n 1 (1983), pp. 43-59; Fragments et collages : essai sur le féminisme québécois des années 70 , Ville Saint-Laurent, les Éditions du Remue-Ménage, 1986, 168 p. ; L'amère patrie. Féminisme et nationalisme dans le Québec contemporain, Montréal, Éditions du Remue-ménage, 2001, $181 \mathrm{p}$. Une des études les plus importantes sur ce sujet est la thèse de maitrise inédite mais largement diffusée de Stéphanie Lanthier sur l'imagerie sexuelle dans le mouvement nationaliste québécois, L'impossible réciprocité des rapports politiques entre le nationalisme radical et le féminisme radical au Québec 1961-1972, Thèse de maitrise (histoire), Université de Sherbrooke, 1998, 127 p. La volonté de Susan Mann de prendre au sérieux le nationalisme québécois et les importantes réflexions qu'elle a tôt formulées sur le nationalisme et le féminisme font qu'elle n'obéit pas à la séparation habituelle entre historiens anglophones et francophones au Canada. Voir, par exemple, Susan Mann Trofimenkoff, « Nationalism, Feminism \& Canadian Intellectual History », Canadian Literature, vol. 83 (hiver 1979), pp. 7-20. On trouvera une importante collection d'articles présentant en détail la seconde vague du mouvement féministe dans Constance Backhouse et David Flaherty dir., Challenging 
Times: The Women's Movement in Canada and the United States, Montréal et Kingston, McGill-Queen's University Press, 1992, 335 p. À l'exception notable de Micheline de Sève et Micheline Dumont, peu d'auteurs parlent de l'importance du nationalisme dans la constitution du mouvement féministe. La Revue d'études canadiennes, dans un numéro spécial consacré à un examen de la relation entre féminisme et nationalisme au Canada, montre toutefois que les féministes canadiennes-anglaises commencent à prendre le nationalisme au sérieux. Voir, par exemple, Jill Vickers, "Feminisms and Nationalisms in English Canada », Revue d'études canadiennes, vol. 35, n² 2, (été 2000), pp. 128148 ; Shauna Wilton, « Manitoba Women nurturing the Nation: The Manitoba IODE and Maternal Nationalism 1913-1920», Revue d'etudes canadiennes, vol. 35, n 2, (été 2000), pp. 145-169 ; Sylvia Bashevkin, «In the Shadow of Free Trade: Nationalism, Feminism and Identity Politics in Contemporary English Canada », Revue d'études canadiennes, vol. 35, n² 2, (été 2000), pp. 109 127. La relation entre nationalisme et féminisme a aussi été l'objet de certains ouvrages internationaux très stimulants. Voici quelques-uns des titres étrangers les plus stimulants : George Moss, Nationalism and Sexuality: Middle Class Morality and Sexual norms in Modern Europe. Madison, University of Wisconsin Press, 1988. 232 p. ; A. Parker, dir. Nationalisms and Sexualities. New York, Routledge, 1992, 232 p. En outre, l'édition de l'hiver 1995 du Journal of Women's History présente de nombreuses réflexions intéressantes sur la relation entre nationalisme et féminisme. Nous avons trouvé particulièrement utiles les arguments d'Ida Blom quant à la nécessité d'examiner le contexte culturel. Ida Blom, "Feminism and Nationalism in the Early Twentieth Century: A cross-cultural Perspective », Journal of Women's History, vol. 7, $\mathrm{n}^{\circ} 4$, (hiver 1995), pp. 82-94.

${ }^{2}$ Micheline de Sève, «Féminisme et nationalisme au Québec, une alliance inattendue ", Revue internationale d'études canadiennes, 17 (printemps 1998), p. 159.

${ }^{3}$ Violette Brodeur, Suzanne G. Chartrand, Louise Corriveau, Béatrice Valay, Le Mouvement des femmes au Québec : études des groupes montréalais et nationaux, Montréal, Centre de formation populaire, 1982, p. 27.

${ }^{4}$ Micheline Dumont, "The Origins of the Women's Movement in Québec », dans Constance Backhouse et David Flaherty dir. op. cit., p. 86.

${ }^{5}$ Paul-André Linteau, René Durocher, Jean-Claude Robert, François Ricard, Histoire du Québec Contemporain : Le Québec depuis 1930, Montréal, Boréal Compact, 1989, tome II, p. 612. Pour une discussion sur les groupes féministes précédents cette époque au Québec, voir Le Collectif Clio, L'Histoire desfemmes au Québec depuis quatre siècles, Montréal, Les Quinze, 1982, 521 p. 
${ }^{6}$ Violette Brodeur, Suzanne G. Chartrand, Louise Corriveau, Béatrice Valay, op. cit., p. 29.

${ }^{7}$ Solange Chalvin, «Le Front commun des Québecoises descendra dans la rue, ce soir », Le Devoir, (28 novembre 1969).

${ }^{8}$ Voir Alice Echols, Daring to Be Bad: Radical Feminism in America 1967-1975, Minneapolis, University of Minnesota Press, 1989, 416 p.

${ }^{9}$ Martine Lanctôt, La genèse et l'evolution du mouvement de libération des femmes à Montréal, 1969-1979, thèse de maitrise (histoire), UQAM, 1980, p. 56.

10 -.--, « Revolution in the Revolution: second manifesto by a collective of women in the Front de Liberation des Femmes Québecoises », Montréal, septembre 1971, Canadian Women's Movement Archives, Boîte 31. Pour un examen stimulant de luttes antérieures de femmes au sein d'organisations de gauche, voir Joan Sangster, Dreams of Equality: Women On The Canadian Left, 1920-1950, Toronto, McClelland and Stewart, 1989, 273 p.

${ }^{11}$ Stéphanie Lanthier, op. cit., résumé.

12 -.--, « Revolution in the Revolution », loc. cit.

13 ----, « F.L.F.Q. Fonctionnement ", été 1970, dans Véronique O’Leary et Louse Toupin dir. Québécoises deboutte!, Ville Saint-Laurent, Éditions du Remue-Ménage, 1982, tome I [ci-après $Q D I$ ], pp. 66-67.

${ }^{14}$ Véronique O'Leary, «Nous sommes le produit d'un contexte», QDI, p. 47.

${ }^{15} \mathrm{FLF}$, «Bulletin de Liaison FLFQ», n 2 (août 1971), dans QDI, p. 117.

${ }^{16}$ Véronique O’Leary, «Nous sommes le produit d'un contexte», QDI, p. 32.

${ }^{17}$ Stéphanie Lanthier, op. cit., pp. 54, 58.

${ }^{18}$ FLF, «FLFQ : Historique », été 1970, dans QDI, p. 66.

${ }^{19} \mathrm{FLF}$, «Bulletin de Liaison FLFQ - cellule journal », $\ln ^{\circ} 2$ (août 1971), dans QDI, p. 115.

${ }^{20}$ Véronique O'Leary, loc. cit., p. 27.

${ }^{21}$ Diane Lamoureux, loc. cit., pp. 44, 46.

${ }^{22}$ FLF, «Bulletin de Liaison FLFQ», n 2 (août 1971), dans QDI, p. 116.

${ }^{23}$ Diane Lamoureux, loc. cit., p. 47.

${ }^{24} \mathrm{FLF}$, «Bulletin de Liaison FLFQ», n 2 (août 1971), dans QDI, p. 117. 
${ }^{25}$ Idem, p. 119.

${ }^{26} \mathrm{FLF}$, « communiqué de presse », 8 mai 1970, Montréal, dans QDI, p. 71.

${ }^{27}$ Lanctôt, op. cit., p. 61.

${ }^{28}$ Idem, p. 60.

${ }^{29}$ Véronique O'Leary et Louise Toupin, «Un bilan de parcours », dans QDI, pp. 76-77. Il est intéressant de noter que, dans la table ronde créée pour discuter du FLF en 1982, aucune des anglophones fondatrices n'était présente, et que la décision d'exclure les anglophones n'a pas été abordée (sauf par Francine Aubin, qui a simplement déclaré avoir adhéré au FLF lors du débat sur l'exclusion en septembre 1970).

${ }^{30} \mathrm{FLF}$, «Lettre à des féministes américaines », 4 décembre 1970, dans QDI, pp. 79-80.

${ }^{31}$ Le Collectif Clio, op. cit., p. 482.

${ }^{32}$ D'après deux anciennes militantes, " au Front de libération des femmes, avait "succédé" le Centre des femmes, formé en partie d'ex-militantes de ce groupe. » Véronique O’Leary et Louise Toupin, «Un outil pour les féministes", dans Véronique O’Leary et Louise Toupin dir. Québécoises deboutte!, Ville Saint-Laurent, Éditions du Remue-Ménage, 1982, tome II [ci-après QDII], p. 8.

${ }^{33}$ Lanctôt, op. cit., p. 87.

34 -..--, «lettre à nos camarades », Québécoises deboutte !, vol. 1, n 1, (novembre 1972), dans QDII, p. 18.

35 -.---, « Histoire d'une oppression ", Québécoises deboutte!, vol. 1, n 2, (décembre 1972), dans QDII, p. 41

36 -----, « Histoire d'une oppression », Québécoises deboutte !, vol. 1, n 1, (novembre 1972), dans $Q D I I$, p. 28.

${ }^{37}$ Idem.

38 --.--, «pour un mouvement de femmes : mais lequel ? », Québécoises deboutte !, vol. 1, n 4, (mars 1973), dans QDII, p. 95.

${ }^{39}$ Idem, p. 94.

${ }^{40}$ Diane Lamoureux, "La lutte pour le droit à l'avortement, 1969-1981 ", Revue d'bistoire de l'Amérique française, vol. 37, $\mathrm{n}^{\circ}$ 1, (juin 1983), p. 82.

${ }^{41}$ Cité dans Idem, p. 84. 
42 -.---, «L'affaire Morgentaler », Québécoises deboutte!, vol. 1, n 5, (avril 1973), dans QDII, p. 163.

43 -----, «Les intérêts en cause », Québécoises Deboutie !, vol. 1, n 7, (juillet-août 1973), dans QDII, p. 220.

44 -.---, «l'avortement, une bataille politique », Québécoises Deboutte !, vol. 1, $\mathrm{n}^{\circ}$ 7, (juillet-août 1973), dans QDII, p. 225.

45 -----, « Problème politique : lutte politique ", Québécoises Deboutte !, vol. 1, $\mathrm{n}^{\circ} 7$, (juillet-août 1973), dans QDII, pp. 225-226. Il faut toutefois noter que cette décision a fait l'objet de beaucoup de discussions et de divisions au sein du Centre des femmes. Voir : -.-.. «Bilan du Centre des Femmes à Montréal, janvier 1972 à septembre 1974 », novembre 1974, dans QDI, pp. 156-174.

46 -.---, « Problème politique : lutte politique », Québécoises Deboutte !, vol. 1, $\mathrm{n}^{\circ}$ 7, (juillet-août 1973), dans QDII, p. 225.

${ }^{47}$ Lamoureux, « La lutte pour le droit à l'avortement, 1969-1981 », loc. cit., p. 84.

${ }^{48}$ Linteau et al., op. cit., p. 685.

${ }^{49}$ Pierre Vallières, Les Héritiers de Papineau. Itinéraire politique d'un "nègre blanc 》 (1960-1985), Montréal, Québec/Amérique, 1986, p. 229.

${ }^{50}$ Voir Ian McKay, « For a New Kind of History: A Reconnaissance of 100 Years of Canadian Socialism ", Labour / Le Travail, vol. 46 (automne 2000), p. 121. Pour une discussion du rôle actif d'une aile de gauche dans le $P Q$ après 1972, voir Richard Jones, «L'Idéologie du Parti Québécois », dans Fernand Dumont dir., Idéologies au Canada Français, 1940-1976, Québec, Les Presses de l'Université Laval, 1981, tome II, p. 237.

${ }^{51}$ Voir Charles Gagnon, Pour le parti prolétarien, Montréal, Equipe du Journal, 1972, 49 p. Dans cet essai, Gagnon parle abondamment du « cul-de-sac nationaliste », affirmant que "Avec les sociaux-démocrates, les nationalistes petits-bourgeois composent le courant idéologique présentement le plus dangereux au sein du mouvement ouvrier. » (p. 11).

${ }^{52}$ Voir Gilles Bourque et Gilles Dostaler, Socialisme et indépendance, Montréal, Boréal Express, 1980, p. 19 ; Louis Fournier, F.L. . The anatomy of an Underground Movement translated by Edward Baxter, Toronto, NC Press Limited, 1984, p. 335. Aussi, voir Jacques Benoît, L'extrême gauche, Ottawa, La Presse Ltée, 1987, 137 p. Pour une discussion du rôle actif d'une aile de gauche dans le PQ après 1972, voir Richard Jones, «L'Idéologie du Parti Québécois », 
dans Fernand Dumont dir., Idéologies au Canada Français, 1940-1976, Québec, Les Presses de l’Université Laval, 1981, tome II, p. 237.

${ }^{53}$ Un bon exemple de cette ambiguité est la manière dont le PQ a réagi au mouvement des «Yvettes » lors du référendum de 1980. Cet incident, selon Diane Lamoureux, « permet de mettre en lumière l'ambiguité du discours péquiste en ce qui concerne les femmes ». Lamoureux, loc. cit., p. 56.

${ }^{54}$ Idem, pp. 51-52.

55 -----, « Le P.Q. espoir ou illusion », Québécoises deboutte!, vol 1, n 5, (avril 1973), dans QDII, pp. 127-128.

${ }^{56}$ Idem, p. 130.

57 -----, «Sur notre condition : un salaire à la ménagère ?», Québécoises deboutte !, vol. 1, n 5, (avril 1973), dans QDII, p. 134.

58 -.---, « Pour une vraie fête des mères \& ménagères », Québécoises deboutte!, vol. 1, nº 6, (juin 1973), dans QDII, p. 171.

59 -----, « A la regent knitting... », Québécoises deboutte !, vol. 1, n 3, (février 1973), dans QDII, p. 71.

${ }^{60}$-----, « Sur notre condition : les ménagères », Québécoises deboutte!, vol. 1, $\mathrm{n}^{\circ}$ 4, (mars 1973), dans QDII, pp. 109, 113.

${ }^{61}$-----, « Bilan du Centre des Femmes à Montréal, janvier 1972 à septembre $1974 »$, novembre 1974, dans QDI, pp. 156-157.

62 ..---, «La journée internationale des femmes », Québécoises deboutte!, vol. 1, n 9, (octobre 1973), dans QDII, p. 307.

${ }^{63}$-.--.. «Les femmes en Chine ». Québécoises Deboutte !, vol. 1, n 7, (juilletaoût 1973), dans QDII, pp. 249-256.

${ }^{64}$-.---, « «encontre avec deux féministes marxistes », Québécoises deboutte!, vol. 1, nº 6, (juin 1973), dans QDII, pp. 190-203.

65 -.--, « Bilan du Centre des Femmes à Montréal, janvier 1972 à septembre $1974 »$, novembre 1974, dans QDI, p. 157.

${ }^{66} \mathrm{Idem}$.

${ }^{67}$ Le Collectif Clio, op. cit., p. 493.

${ }^{68}$ «Table ronde : Front de libération des femmes du Québec, le 16 novembre 1982 », dans QDII, p. 337. 